\title{
The Ultra-Fast Flash Observatory's space GRB mission and science
}

H. Lim ${ }^{1}$, S. Ahmad ${ }^{2}$, P. Barrillon ${ }^{2}$, S. Blin-Bondil ${ }^{2}$, S. Brandt ${ }^{3}$, C. ${\text { Budtz-J } \varnothing \text { rgensen }^{3} \text { A. J. Castro-Tirado }{ }^{4}, \text { P. Chen }}^{5}$, H. S. Choi ${ }^{6}$, Y. J. $\mathrm{Choi}^{7}$, P. Connell ${ }^{8}$, S. Dagoret-Campagne ${ }^{2}$, C. De La Taille ${ }^{2}$, C. Eyles $^{8}$, B. Grossan ${ }^{9}$, I. Hermann ${ }^{7}$, M. -H. A. Huang ${ }^{10}$, S. Jeong ${ }^{1}$, A. Jung $^{1}$, J. E. Kim ${ }^{1}$, S. -W. Kim ${ }^{3}$, Y. W. Kim ${ }^{1}$, J. Lee ${ }^{1}$, E. V. Linder ${ }^{1,9}$, T. -C. $\mathrm{Liu}^{5}$, N. Lund ${ }^{3}$, K. W. Min ${ }^{7}$, G. W. Na ${ }^{1}$, J. W. Nam ${ }^{1}$, K. H. Nam $^{1}$, M. I. Panasyuk ${ }^{12}$, I. H. Park ${ }^{1}$, V. Reglero ${ }^{8}$, J. Ŕípa ${ }^{1}$, J. M.

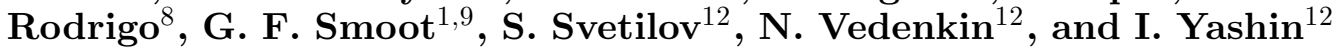

\author{
${ }^{1}$ Ewha Womans University, Seoul, Korea \\ email: heuijin.lim@gmail.com \\ ${ }^{2}$ University of Paris-Sud 11, France \\ ${ }^{3}$ National Space Institute, Denmark \\ ${ }^{4}$ Instituto de Astrofisica de Andalucia, CSIC, Spain \\ ${ }^{5}$ National Taiwan University, Taipei, Taiwan \\ ${ }^{6}$ Korea Institute of Industrial Technology, Ansan, Korea \\ ${ }^{7}$ Korea Advanced Institute of Science and Technology, Daejeon, Korea \\ ${ }^{8}$ University of Valencia, Spain \\ ${ }^{9}$ University of California, Berkeley, USA \\ ${ }^{10}$ National United University, Miao-Li, Taiwan \\ ${ }^{11}$ Yonsei University, Seoul, Korea \\ ${ }^{12}$ Moscow State Univ., Moscow, Russia
}

\begin{abstract}
The Ultra-Fast Flash Observatory (UFFO) is a space mission to detect the early moments of an explosion from Gamma-ray bursts (GRBs), thus enhancing our understanding of the GRB mechanism. It consists of the UFFO Burst \& Trigger telescope (UBAT) for the recognition of GRB positions using hard X-ray from GRBs. It also contains the Slewing Mirror Telescope (SMT) for the fast detection of UV-optical photons from GRBs. It is designed to begin the UV-optical observations in less than a few seconds after the trigger. The UBAT is based on a coded-mask X-ray camera with a wide field of view (FOV) and is composed of the coded mask, a hopper and a detector module. The SMT has a fast rotatable mirror which allows a fast UV-optical detection after the trigger. The telescope is a modified Ritchey-Chrétien telescope with the aperture size of $10 \mathrm{~cm}$ diameter, and an image intensifier readout by CCD. The UFFO pathfinder is scheduled to launch into orbit on 2012 June by the Lomonosov spacecraft. It is a scaled-down version of UFFO in order to make the first systematic study of early UV/optical light curves, including the rise phase of GRBs. We expect UBAT to trigger $\sim 44$ GRBs/yr and expect SMT to detect $\sim 10 \mathrm{GRBs} / \mathrm{yr}$.
\end{abstract}

Keywords. gamma rays: bursts, gamma rays: observations, instrumentation: detectors

\section{Overview}

The Ultra-Fast Flash Observatory (UFFO) aims to measure the early UV/optical observations of GRBs using the new approach of a beam steerer which can be implemented by a rotatable mirror in the UV/optical telescope. It allows $\mathrm{UV} /$ optical observations to 

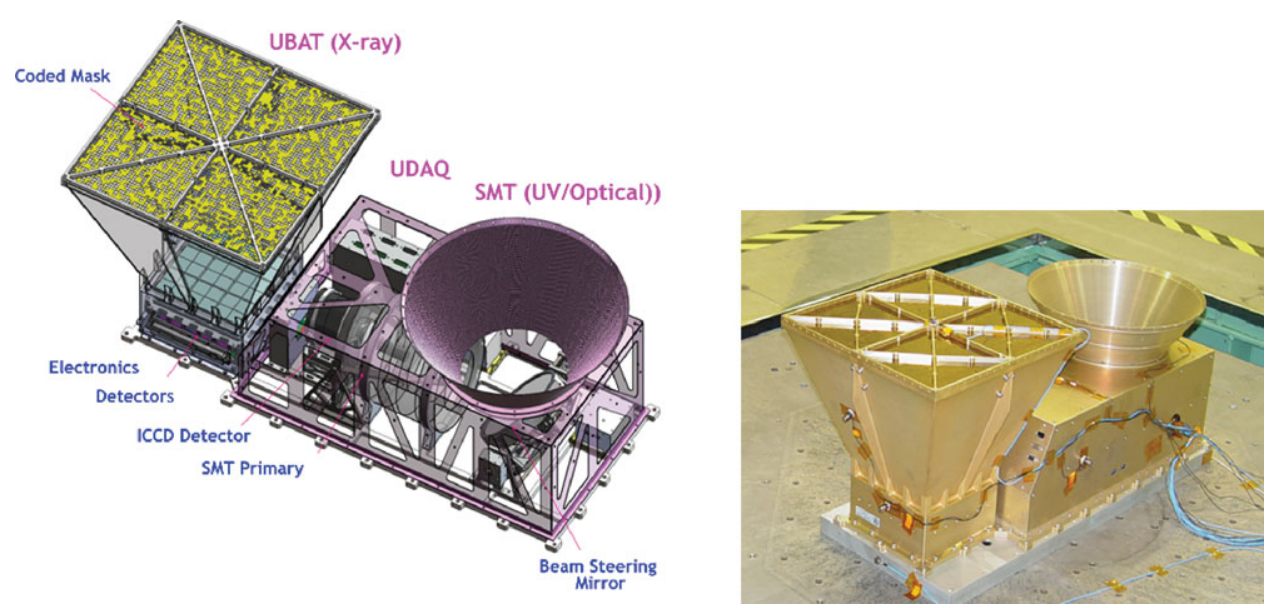

Figure 1. Left: UFFO pathfinder payload. Right: UFFO pathfinder pre-flight model which passed its space environment tests on 2011-July.

begin in less than a few seconds after the X-ray trigger Park et al. 2009). The UFFO will exploit this largely unexplored region of parameter space by providing a statistically significant sample of early UV/optical observations of GRBs.

The UFFO consists of a couple of wide Field-of-View (FOV) trigger telescopes, a narrow-FOV Slewing Mirror Telescope (SMT) for the fast measurement of the UV/optical photons from GRBs, and a gamma-ray monitor for energy measurement. The UFFO Burst Alert \& Trigger Telescope (UBAT) will provide the primary trigger using X-rays from GRBs. It monitors the sky for GRBs and determines their position with sufficient accuracy $(10$ arcmin at $7.0 \sigma)$ for follow-up in the UV/optical with the SMT. Whereas the fastest previous experiment, the Swift observatory, rarely observed GRBs in less than 60 seconds after the trigger, the UFFO is designed to begin UV/optical observations in less than a few seconds after the trigger. The SMT uses the novel approach of steering our telescope beam using a rotatable mirror, instead of re-orienting the instrument platform like Swift and other previous instruments. The UFFO pathfinder is the scaled-down version of UFFO with a physical size of $958.5(\mathrm{~L}) \times 400(\mathrm{~W}) \times 382.5(\mathrm{H}) \mathrm{mm}^{3}$ (See Fig. 1(left)) and is scheduled to launch into orbit on 2012-June by the Lomonosov spacecraft. It will be on a sun-synchronous orbit at an altitude of $\sim 550 \mathrm{~km}$. It successfully passed the thermal-vacuum test and the vibration-shock test on 2011-July at Taiwan NSPO (See Fig. 1(right)) and it is in the final stage for launch preparations.

\section{Acknowledgements}

This research was supported by the Basic Science Research Program through the National Research Foundation of Korea (NRF) that is funded by the Ministry of Education, Science and Technology (2010-0025056). This research is also supported by the World Class University (WCU) program through the NRF that is funded by the Ministry of Education, Science and Technology (R32-2008-000-10130-0) in Korea.

\section{Reference}

Park, I. H., et al. 2009, arXiv:0912.0773 